\title{
Características de las gestantes que recibieron Psicoprofilaxis Obstétrica de Emergencia en el sector privado como una alternativa en su atención integral
}

Sabrina Morales Alvarado ${ }^{1,2}$

\section{RESUMEN}

El obj etivo de este estudio fue conocer las características de un grupo de gestantes del sub sector privado que recibieron Psicoprofilaxis obstétrica de Emergencia (PPO-EM) entre Julio 2012 y junio 2014, asimismo, conocer las razones por las que no realizaron su preparación prenatal integral oportunamente, es decir, entre el quinto y séptimo mes, de acuerdo a las recomendaciones técnicas generales. Se revisaron y evaluaron 168 historias clínicas. Los rangos de edad fueron de 15 a 45 años (promedio: 30), el grado de instrucción se distribuyó en: secundaria incompleta $0.6 \%$, secundaria completa $6 \%$, superior incompleta $15.4 \%$ y superior completa $78 \%$. El $69 \%$ se encontraba trabajando durante su gestación. No recibieron PPO por falta de: información el $17.3 \%$, tiempo $46.4 \%$, recursos económicos $28.6 \%$ y por falta de apoyo de la pareja 7.7\%. El 100\% manifestó satisfacción sobre su PPO-EM, por sentirse más tranquilas y seguras de cómo llegar y colaborar en su parto. Finalmente, 94.6\% recibió PPO-EM en la última semana de gestación y $5.4 \%$ durante su trabajo de parto; $64 \%$ tuvo parto vaginal y el resto cesárea.

Se concluye que la PPO-EM es una oportunidad beneficiosa para las gestantes que no pudieron realizar PPO a tiempo, por lo que se afirma que nunca es tarde para recibir una buena preparación y apoyo integral, los que se asociaron a un buen resultado materno y perinatal. (Horiz Med 2015; 15(1): 61-66)

Palabras clave: Psicoprofilaxis obstétrica, emergencia, gestante, parturienta, recién nacido, equipo de salud. (Fuente: DeCS BIREME).

Characteristics of pregnant who received Emergency Obstetric Psychoprophylaxis in private sector as an alternative to comprehensive care

\section{ABSTRACT}

The aim of this study was to determine the characteristics of a group of pregnant women in sub private sector who received Emergency Obstetric Psychoprophylaxis (EM-OPP) between July 2012 and June 2014, also know the reasons why they did not perform their integral prenatal preparation to timely gestational age, between the fifth and seventh month, according to general technical recommendations, age ranges and educational level. Were reviewed and assessed 168 medical records, noting that the age range was 15-45 years (mean: 30 ), educational attainment was distributed: incomplete secondary $0.6 \%$, complete secondary $6 \%$ incomplete higher $15.4 \%$ and above $78 \%$ complete. According to their employment status, $69 \%$ were working during pregnancy. Regarding his prenatal emergency preparedness, it was found that $100 \%$ of mothers reported satisfaction in receiving EM-OPP, to feel calmer and safer to directions and contribute to its delivery. Finally, 94.6\% received their PPO-EM during the last week of gestation while $5.4 \%$ during labor, and the type of delivery, $64 \%$ ended in vaginal birth and the rest via cesarean section. We conclude that the PPO-EM is a beneficial opportunity for pregnant women who were unable to make timely prenatal preparation, which states that it is never too late to get a good preparation, which was associated with a good perinatal outcome. (Horiz Med 2015; 15(1): 61-66)

Key words: Obstetric psychoprophylaxis, emergency, pregnant, newborn, health team. (Source: MeSH NLM).

Obstetra. Doctora en Ciencias de la Salud.

Directora de la Escuela Académico Profesional de Obstetricia - Universidad Norbert Wiener. Lima -Perú 


\section{INTRODUCCIÓN}

La atención de una gestante, se considera completa cuando además del control clínico periódico, recibe oportunamente una preparación prenatal integral, a través de la Psicoprofilaxis Obstétrica (PPO), sin embargo, pueden haber factores por los cuales no la reciba a tiempo, por lo que al acercarse la semana de la fecha probable de parto, se hace muy difícil lograr que todos los componentes de la PPO (preparación teórica, física y psico-afectiva), logren todos los contenidos (1); no obstante ello, la gestante puede tener la oportunidad de recibir una orientación y entrenamiento centralizado en lo más importante que necesita para lograr los principales objetivos de esta atención, como la tranquilidad, seguridad y habilidades para aplicar técnicas de autoayuda que incluso serán muy beneficiosas para ella y el bebé durante su trabajo de parto, es decir, a través de una Psicoprofilaxis Obstétrica de Emergencia (PPO$E M)$, definición que fue propuesta en 1995 por la autora del presente estudio, a la Sociedad Peruana de Psicoprofilaxis Obstétrica (ASPPO), la cual luego de su aceptación fue instaurada, siendo esencia en diversas publicaciones como el Manual de Psicoprofilaxis Obstétrica ASPPO desde su primera edición en 1997 (2).

El presente estudio, refleja la importancia y posibilidad que tiene la gestante 0 parturienta de recibir una PPO-EM, dado que, con ello puede mejorar su condición física y psico-afectiva, logrando una mejor respuesta y calidad de vida, al contar con instrumentos de información y autoayuda que le permitirá sentirse más tranquila y dispuesta a colaborar con el nacimiento de su hijo.

\section{EL ESTUDIO}

Se realizó un estudio observacional, descriptivo y transversal, con 168 historias clínicas de pacientes gestantes y parturientas del sector privado que recibieron Psicoprofilaxis Obstétrica de emergencia.

Se analizó cada historia clínica incluyéndose las siguientes variables: edad, grado de instrucción, condición laboral, motivo por el que no iniciaron oportunamente la Psicoprofilaxis Obstétrica, motivo por el que buscaron la PPO-EM, momento en que recibieron la PPO-EM y el tipo de parto y satisfacción de las madres al haber recibido PPOEM.

Se elaboró una plantilla en Excel con los detalles respectivos, lista para ser Ilenada de acuerdo a los hallazgos de cada historia clínica.

\section{HALLAZGOS}

El rango de edad fue 15 a 45 años, siendo la moda de 31 a 40 años. Distribuyéndose de la siguiente manera: Entre 15 y 19 años 11 (6.6\%), entre 20 a 30 años 42 (25\%), de 31 a 40 años 79 (47\%) y mayores de 40 años 36 (21.4\%), Tabla 1.

Tabla 1. Rango de edad de las gestantes que recibieron ppo-em

\begin{tabular}{l|c|c} 
Edad en años & $\mathbf{N}^{\circ}$ & $\%$ \\
\hline $15-19$ & 11 & 6.6 \\
\hline $20-30$ & 42 & 25 \\
\hline $31-40$ & 79 & 47 \\
\hline$>40$ & 36 & 21.4 \\
\hline TOTAL & 168 & 100 \\
\hline
\end{tabular}

La distribución según el grado de instrucción fue: secundaria incompleta $1(0.6 \%)$, secundaria completa $10(6 \%)$, superior incompleta $26(15.4 \%)$ y superior completa 131 (78\%), Tabla 2.

Tabla 2. Distribución de las gestantes que recibieron PPO-EM de acuerdo al grado de instrucción

\begin{tabular}{|l|c|c|}
\hline Grado de Instrucción & $\mathbf{N}^{\circ}$ & $\%$ \\
\hline Secundaria incompleta & 1 & 0.6 \\
\hline Secundaria completa & 10 & 6 \\
\hline Superior incompleta & 26 & 15.4 \\
\hline Superior completa & 131 & 78 \\
\hline TOTAL & $\mathbf{1 6 8}$ & $\mathbf{1 0 0}$
\end{tabular}

Según su condición laboral: se encontraban trabajando 116 (69\%), mientras que 52 (31\%) no estaban laborando, Tabla 3.

Tabla 3. Distribución de las gestantes de acuerdo a su condición laboral

\begin{tabular}{|l|c|c|}
\hline Condición laboral & $\mathbf{N}^{\circ}$ & $\%$ \\
\hline Con trabajo & 116 & 69 \\
\hline Sin trabajo & 52 & 31 \\
\hline TOTAL & 168 & 100 \\
\hline
\end{tabular}


De acuerdo al motivo por el que no iniciaron oportunamente la PPO: Por falta de información sobre la importancia de la PPO 29 (17.3\%), por falta de tiempo $78(46.4 \%)$, por falta de recursos económicos $48(28.6 \%)$ y por falta de apoyo de la pareja $13(7.7 \%)$, Tabla 4.

Tabla 4. motivo por el cual la paciente no inició la PPO oportunamente

\begin{tabular}{l|c|c}
\hline Motivo & $\mathbf{N}^{\circ}$ & $\%$ \\
\hline $\begin{array}{l}\text { Falta de información sobre la importancia de la } \\
\text { PPO }\end{array}$ & 29 & 17.3 \\
\hline Falta de tiempo & 78 & 46.4 \\
\hline Falta de recursos económicos & 48 & 28.6 \\
\hline Falta de apoyo de la pareja & 13 & 7.7 \\
\hline TOTAL & 168 & 100 \\
\hline
\end{tabular}

Sobre el motivo por el cual la paciente decidió buscar PPO-EM se encontró que: Por temor a "última hora" $101(60 \%)$, por recomendación de un familiar/amistad 53 (32\%) y por recomendación de un profesional de la salud 14 (8\%), Tabla 5.

Tabla 5. Motivo por el cual la paciente decidió buscar PPO-EM

\begin{tabular}{l|c|c}
\hline Motivo & $\mathbf{N}^{\circ}$ & $\%$ \\
\hline Temores de "última hora" & 101 & 60 \\
\hline Recomendación: familiar/amistad & 53 & 32 \\
\hline Recomendación: profesional de la salud & 14 & 8 \\
\hline TOTAL & 168 & 100 \\
\hline
\end{tabular}

Según el momento en que recibieron la PPOEM resultó: Durante la última semana de edad gestacional 159 (94.6\%) y durante el trabajo de parto $9(5.4 \%)$, Tabla 6.

Tabla 6. Distribución según el momento en que se brindó la ppo-em

\begin{tabular}{|l|c|c|}
\hline Momento de la PPO-EM & $\mathbf{N}^{\circ}$ & $\%$ \\
\hline $\begin{array}{l}\text { Durante la última semana de edad } \\
\text { gestacional }\end{array}$ & 159 & 94.6 \\
\hline Durante el trabajo de parto & 9 & 5.4 \\
\hline TOTAL & 168 & 100
\end{tabular}

Según el tipo de parto: Vaginal 107 (64\%) y por cesárea 61 (36\%), Tabla 7.

Tabla 7. Distribución según el tipo de parto

\begin{tabular}{l|c|c|}
\hline Tipo de parto & $\mathbf{N}^{\circ}$ & $\%$ \\
\hline Vaginal & 107 & 64 \\
\hline Cesárea & 61 & 36 \\
\hline TOTAL & 168 & 100 \\
\hline
\end{tabular}

Finalmente, las 168 pacientes (100\%), manifestaron su satisfacción de haber recibido PPO-EM,Tabla 8.

Tabla 8. Satisfacción de las madres por haber recibido PPO-EM

\begin{tabular}{|l|c|c|}
\hline Satisfacción & $\mathbf{N}^{\circ}$ & $\%$ \\
\hline Satisfechas & 168 & 100 \\
\hline No satisfechas & 0 & 0 \\
\hline TOTAL & 168 & 100
\end{tabular}

\section{DISCUSIÓN}

El presente estudio es relevante dado que aporta información sobre la oportunidad que pueden tener las gestantes o parturientas de recibir PPO-EM, en caso de no haber realizado su preparación prenatal a tiempo, independientemente de los motivos, con lo cual se afirma que toda madre tiene el deber, derecho y oportunidad de recibir preparación y apoyo psico-afectivo antes o durante su trabajo de parto, independiente a su edad gestacional (3).

Por definición, la Psicoprofilaxis de emergencia (PPO-EM), es la preparación brindada a la gestante faltando pocos días para la fecha probable de parto, o la que se ofrece a la parturienta durante su labor de parto.

Es importante la adaptación de un programa 0 sesión intensiva teniendo en cuenta las prioridades.

Busca esencialmente la tranquilidad, colaboración y participación de la gestante/ parturienta para un mejor control emocional. La principal herramienta de la PPO-EM, proviene de la escuela soviética ya que busca el "automatismo de la respuesta condicionada" para facilitar una mejor actitud, rol activo y experiencia obstétrica en la madre (4).

La aplicación de la PPO-EM sea a una gestante o parturienta, considera el importante rol y participación del padre (Figura 1).

Asimismo, toma en cuenta los tres pilares de la PPO (información, entrenamiento y apoyo psicoafectivo), los que según el tiempo y necesidades de la madre, se priorizan para lograr los principales objetivos de acuerdo a su definición, utilizando métodos y técnicas básicas y complementarias como 
la relajación, respiración, visualización, masajes localizados, posiciones antálgicas, aromaterapia, sofrología, esferodinamia y musicoterapia, entre otros con la finalidad de mejorar el confort, motivación y entrenamiento de la madre $(5,6)$.

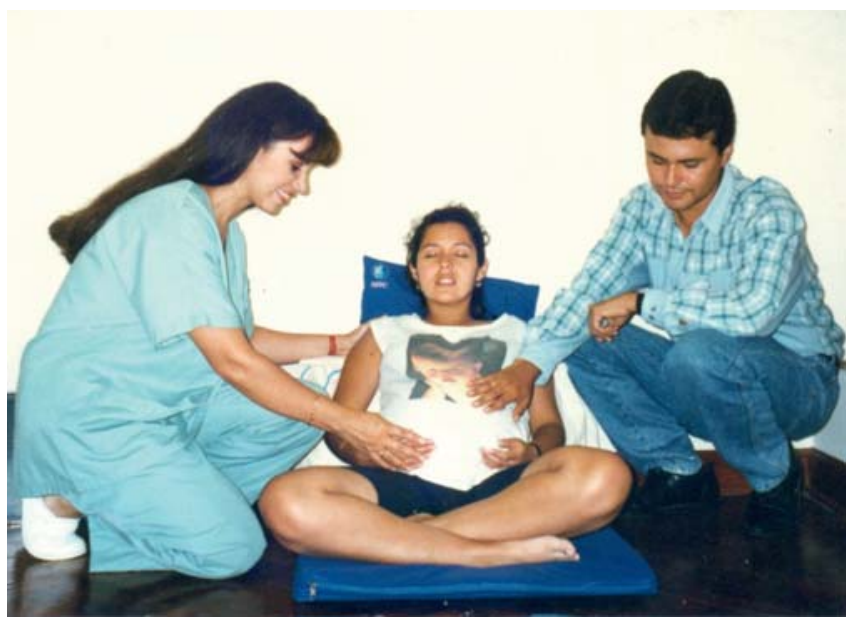

Figura 1. PPO-EM considera el importante rol y participación del padre

Observamos en el estudio, que el rango de edad fue 15 a 45 años y la mayoría de madres que recibieron PPO-EM tenían entre 31 y 40 años, estos datos se asociaron a la mayor frecuencia con el grado de instrucción superior completa y con actividad laboral, lo cual explicaría que a mayor condición educativa y posibilidad laboral, habría mejor oportunidad para que la gestante reciba información y considere la importancia y beneficios que le puede brindar una buena preparación y apoyo psico-afectivo para el nacimiento de su bebé.

Asimismo, cabe mencionar que el grupo significativo de gestantes mayores de 40 años (21.4\%), probablemente se deba a que estuvo conformado por gestantes post procedimiento de fertilización, lo cual cada vez va incrementando el número de gestantes añosas, y en otros casos por la decisión de una maternidad postergada $(7,8)$.

Si bien el grupo con menor frecuencia fue el de adolescentes $(n=11)$, esto permitió objetivar que independientemente a la edad o grado de instrucción, todas las gestantes que no recibieron oportunamente PPO, también pueden beneficiarse con la PPO-EM (9).
Otro aspecto interesante, es respecto del motivo por el que las pacientes no iniciaron oportunamente su PPO, la gran mayoría refirió por falta de tiempo, lo cual estuvo asociado a la condición laboral, seguido por la falta de recursos económicos.

Esto es interesante de considerar, pues significaría, que aun cuando la mayoría de gestantes están informadas de los beneficios de la PPO, no cuentan con las facilidades laborales o económicas para realizar su preparación prenatal.

Es interesante saber que la falta de información sobre la importancia de la PPO (17.3\%), fue de las menores causas de no haber hecho PPO, seguido por la falta de apoyo de la pareja (7.7\%), lo que indicaría que aún existe necesidad de difundir información sobre la importancia y beneficios de la PPO hacia la comunidad en general $(10,11)$.

Es de notar que el motivo principal por el que la mayoría de pacientes decidió buscar PPO-EM fue por temores de "última hora" (60\%), lo que significa que en la generalidad de gestantes afloró un miedo latente hacia el parto, el que decidieron mitigar en lo posible con una o más sesiones antes del parto.

En el caso de las madres que recibieron PPO-EM durante su trabajo de parto, fue debido a que no tuvieron tiempo para ninguna sesión prenatal, Io que compensaron con el acompañamiento y ayuda profesional Obstétrica, recibiendo soporte integral durante el trabajo de parto (12).

Otro detalle que llamó la atención, es que el 32\% buscó PPO-EM por recomendación de un familiar o amistad, esto refleja que un buen sector de la comunidad en general, es consciente de lo beneficioso que es llegar preparada al parto.

Finalmente, pero no menos importante, se observó que solo el $8 \%$,recibió la recomendación de un profesional de la salud, de lo que se podría inferir, que quienes realizan el control prenatal, no manejan información sobre los beneficios de la PPO o PPO-EM, lo que llama la atención pues son los más indicados a brindar información oportuna y obligatoria a toda gestante para que pueda realizar su PPO, independiente a su edad gestacional (13). 
Otro aspecto encontrado, es que la gran mayoría de madres recibió PPO-EM durante la última semana de edad gestacional (94.6\%), mientras que solo el 5.4\% durante el trabajo de parto, lo que significaría que nunca es tarde para recibir alguna sesión de PPO-EM, y hay que buscar el tiempo y al profesional competente (14).

Asimismo, observamos que la mayoría de pacientes, $64 \%$, tuvo parto Vaginal y por cesárea el 36\%; encontrándose ésta última cifra por encima de las recomendaciones de la Organización Mundial de la Salud (15\%). No obstante ello, comparativamente ésta cifra es mucho menor de la que registra estadísticamente del promedio de establecimientos privados, lo que concuerda con los beneficios atribuidos a la PPO en diferentes publicaciones y latitudes entre otros como la reducción del tiempo, instrumentación o medicación del trabajo de parto $(15,16)$.

Cabe resaltar que las 168 pacientes (100\%), manifestaron su satisfacción de haber recibido PPO-EM, por sentirse más tranquilas y seguras de cómo llegar y/ o colaborar durante su labor de parto para el buen nacimiento de su bebé.

En conclusión, la PPO-EM es una oportunidad valiosa que brinda una preparación y apoyo psico-afectivo a toda gestante que no tuvo tiempo de realizarla entre los cinco y siete meses de gestación, por lograr en ella tranquilidad, confianza y métodos de autoayuda a la madre, por lo que hay que considerar que nunca es tarde para recibirla $(17,18)$. Figura 2

\section{Principales recomendaciones en la aplicación de la PPO-EM a una parturienta}

- Hablarle en tono cordial y con seguridad.

- Llamarla por su nombre.

- Ganarnos su confianza.

- Evitar decir la palabra dolor.

- Si la parturienta no respira bien o se descontrola, calmarla con frases como: "Sería mejor si respiras así...".

- Nunca hablarle en negativo, ejemplo: "estás respirando mal"

- Mirarla a los ojos.

- Explicarle de forma clara y resumida el proceso del trabajo de parto.

- Ayudarla a utilizar las posiciones antálgicas. Felicitarla, animarla: "que bien lo haces...".

- Brindarle contacto físico, tomarle la mano, secar su frente, siempre con prudencia respecto de su actitud a veces nerviosa 0 descontrolada.

- Utilizar las técnicas de respiración y realizarlas con ella.

- No descuidar su hidratación.

- Enfatizar la fuerza abdominal durante la respiración sostenida "empuje o pujo".

- Ayudarla a que no pierda la concentración.

- Brindar el acompañamiento en equipo con el padre/familiar.

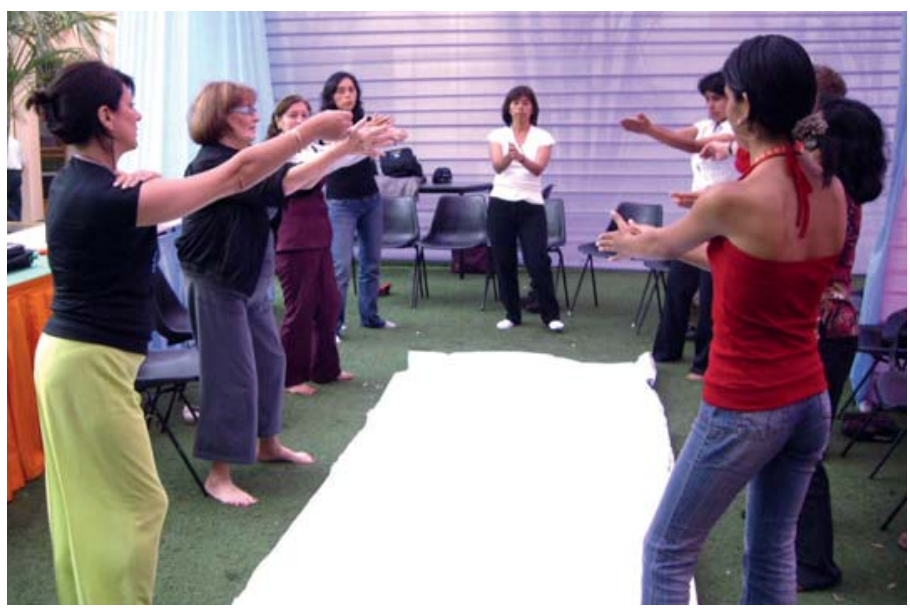

Figura 2. La PPO debe ser oportuna 


\section{REFERENCIAS BIBLIOGRÁFICAS}

1. ASPPO (Soc. Peruana de Psicoprofilaxis Obstétrica). El ABC de la Maternidad y Nacimiento Saludables. Boletín Científico ASPPO. Año $12 \mathrm{~N}^{\circ} 38$.

2. ASPPO (Soc. Peruana de Psicoprofilaxis Obstétrica). Manual de Psicoprofilaxis Obstétrica, 3ra Ed. Lima: ASPPO. 2010.

3. Hospital Bertha Calderón Roque, Centro de Salud Sexual Reproductiva para Adolescentes. Manual de Procedimientos Curso Parto Psicoprofiláctico, $1^{\text {a }}$ Ed. Managua: 2010.

4. Morales S. Psicoprofilaxis Obstétrica: Actualización, definiciones y conceptos. Horiz. Med. vol.14 no.4 Lima oct./dic. 2014.

5. Gavensky R. Parto sin temor y parto sin dolor, 17ma. Ed. Buenos Aires: El Ateneo. 1987.

6. Guzmán A. "Parto psicoprofiláctico vs. Atención obstétrica tradicional".Ginecología y Obstetricia de México 1983; 51(316): 221- 224.

7. Videla M, Grieco A. Parir y Nacer en el Hospital. 1ra Ed. Buenos Aires: Nueva Visión; 1993.

8. Paredes N. Maternidad postergada. Horiz Med 2013; 13(1): 45-50.

9. Yabar M. La Psicoprofilaxis Obstétrica en gestantes adolescentes: Características y beneficios integrales. Horiz. Med. 2014;14(2): 39 . 44

10. Morales S. Psicoprofilaxis obstétrica con ciencia y calidad. Boletín Científico ASPPO 2012; 13(39): 4-5.

11. Ministerio de Salud y Ambiente de la Nación. Preparación integral para la maternidad. Argentina. 2005.

12. Callata Y, Zegarra S. Percepción de los factores limitantes en la atención delparto con acompañante en puérperas atendidas en el Instituto Nacional Materno Perinatal, 2011.

13. Morales S. Impacto de la Psicoprofilaxis Obstétrica en la reducción de la morbilidad y mortalidad materna y perinatal. Horiz Med 2012; 12(2): 47-50.

14. Morales S. Psicoprofilaxis Obstétrica - Guía práctica, 1ra. Ed. Lima: ASPPO. 2012.

15. CLAP - OPS/OMS. Guías para el continuo de atención de la mujer y el recién nacido. Publicación científica $\mathrm{N}^{\circ}$ 1577. Cap. 3, 2011. 298.CLAP - OPS/OMS. Boletín de Salud Perinatal 2000; 18: 1-44.

16. Martinez J. Impacto del Programa de Educación Maternal sobre la Madre y el Recién nacido. Tesis para optar el grado de Doctor. Granada. 2012.

17. MINSA. Guía técnica para la Psicoprofilaxis Obstétrica y Estimulación prenatal. Lima. 2011.

18. Morales S. La Psicoprofilaxis Obstétrica como eje de la prevención prenatal, $1^{a}$. ed. Lima: Cimagraf. 2004. 176.

\section{AGRADECIMIENTOS}

Mi agradecimiento a las pacientes, por su confianza y disposición, lo que hizo posible este artículo original.

\section{CONTRIBUCIONES DE AUTORÍA}

Este fue un estudio donde la autora participó en la recolección, análisis de datos y el proceso de desarrollo del manuscrito.

\section{Fuentes de financiamiento}

Este estudio ha sido autofinanciado por la autora.

\section{Conflictos de interés}

La autora declara no tener ningún conflicto de interés.

\section{Correspondencia:}

\section{Sabrina Ynés Morales Alvarado}

Dirección: Av. Javier Prado Oeste 555 Dpto. 1501

Lima 27, Perú.

Teléfono: +(51 1) 421-0979 / 999-096 282

Correo electrónico: sabrynes@gmail.com sabrinamorales@asppo.org s19@wienergroup.com 